Received: 8 March 2018

Accepted: 19 October 2018

Published online: 27 November 2018

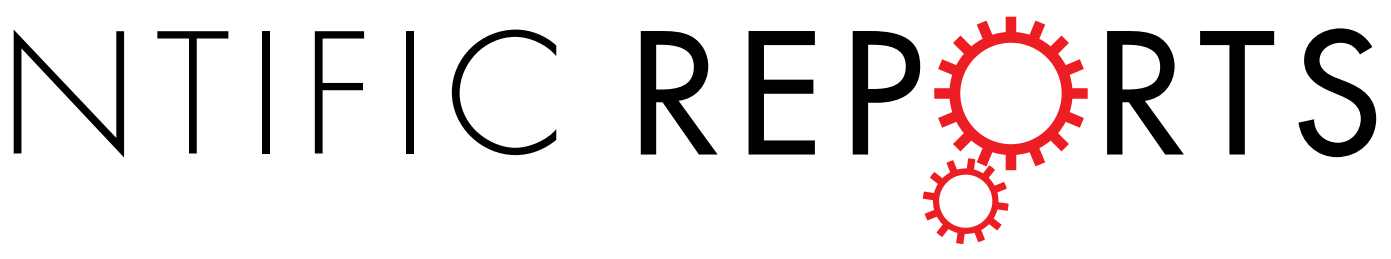

\title{
OPEN Aging of stimulus-driven and goal- directed attentional processes in young immigrants with long-term high altitude exposure in Tibet: An ERP study
}

\author{
Hailin Ma ${ }^{1,2}$, Xiaoyan Huang ${ }^{1}$, Ming Liu ${ }^{1,2,3}$, Huifang Ma ${ }^{4}$ \& Delong Zhang ${ }^{1,2,3}$
}

High altitude (HA) exposure reduces the behavioral response to visual attention and the neural basis is still largely unclear. The present study explored the stimulus-driven and goal-directed factors that are hidden within this attentional behavior impairment via a visual search paradigm in young immigrants in Tibet by recording event-related potential (ERPs). We found that HA explosure significantly slowed the stimulus-driven behaviors instead of the goal-directed behaviors. Furthermore, the P1, N1, and P3 amplitudes collectively indicated the poor efficiency of entire attention behaviors, in which the P3 magnitude of resources allocation was negatively correlated with the attentional behavior response. And the P3 scalp distribution suggested a compensation for insufficient resources of sensory processing only in the goal-directed behaviors. Together, the present study made the point on how stimulus-driven and goal-directed attentional behaviors changed as a result of chronic HA environment exposure, which is similar to aging.

Many studies have indicated behavioral changes under the HA environment, especially motor deterioration and reaction slowness ${ }^{1-4}$. Note that human behaviors generally manifest as the automatic response to external stimuli (stimulus-driven behaviors) and the expression of internal volition (goal-directed behaviors) in the surrounding world ${ }^{5-8}$. And these two points are particularly reflected in attention behaviors. As we noted, visual attention behaviors, such as detecting rapidly threatening stimuli or foraging for your favorite cookies in a crowded supermarket shelves, are very important for the survival of human beings ${ }^{9}$ and are susceptible to the HA exposure ${ }^{3,10,11}$. However, it is still largely unclear how these two points changed with the injure of visual attention behaviors under the HA exposure.

Plateau sections $(2,500 \mathrm{~m}$ above sea level) holding more than 140 million permanent population are mainly distributed in North, Central, and South America, East Africa, and Asia ${ }^{12}$. In China, the Qinghai-Tibetan Plateau is a typical plateau section with hypoxia, low pressure, dry climate, strong ultraviolet rays, thin air, and low temperatures. Of note, there are about 12 million permanent population on the Qinghai-Tibetan Plateau (71.14\% of them live between 2,500 to 4,500 m) as of 2006 and approximately 6 million lowland immigrants ${ }^{2,13,14}$. Moreover, hundreds of thousands of people in lowland areas travel, learn and work, especially engaging in disaster relief and military activity, to the Tibetan plateau every year ${ }^{2}$. Previous studies have discovered the longer time of staying at HA area, the longer the duration of the attention reduction. It would take long time in recovery after people returned to the low altitude (LA) area $^{15}$. And the higher in the altitude place, the less things people can notice $^{16}$. This declining attention performance is claimed to be caused by changes of the functioning neural systems which support attention processes ${ }^{17}$. In many previous studies, the role of attention behavioral processes

${ }^{1}$ Plateau Brain Science Research Center, South China Normal University/Tibet University, Guangzhou, 510631/Lhasa 850012, China. ${ }^{2}$ Center for the Study of Applied Psychology, Key Laboratory of Mental Health and Cognitive Science of Guangdong Province, School of Psychology, South China Normal University, Guangzhou, China. Institute for Brain Research and Rehabilitation, South China Normal University, Guangzhou, 510631, China. ${ }^{4}$ College of Management, Tianjin University, Tianjin, China. Hailin Ma and Xiaoyan Huang contributed equally. Correspondence and requests for materials should be addressed to D.Z. (email: delong.zhang.scnu@gmail.com) 


\begin{tabular}{|l|l|l|l|l|}
\hline \multirow{2}{*}{} & \multicolumn{2}{|l|}{ RTs } & Accuracy \\
\cline { 2 - 5 } & Stimulus-driven & Goal-directed & Stimulus-driven & Goal-directed \\
\hline HA group & $617.44 \pm 134.09$ & $1030.72 \pm 182.67$ & $98.69 \pm 0.01$ & $96.80 \pm 0.02$ \\
\hline LA group & $558.18 \pm 71.92$ & $978.53 \pm 128.88$ & $99.01 \pm 0.01$ & $96.92 \pm 0.02$ \\
\hline
\end{tabular}

Table 1. Averaged RTs (ms) and accurate rates (\%) and their corresponding standard deviations for each of two attention processes (Stimulus-driven attention; Goal-directed attention) in the HA and the LA groups.

including the stimulus-driven behaviors and the goal-directed behaviors was paid more attention, which showed that both were independent ${ }^{18-21}$, suggesting that they had different neural pathways ${ }^{5,22,23}$. However, more recent studies emphasized the relationship of both attention behaviors ${ }^{24}$ in which how the stimulus-driven behaviors competed with the goal-directed behaviors when they mutually coordinated attention behaviors ${ }^{25}$. More details, the stimulus-driven behaviors won the competition ${ }^{24}$, as indicated by more rapid orientation and greater accuracy in the stimulus-driven behaviors ${ }^{26-28}$. Conversely, the stimulus-driven behaviors could be prevented but the goal-directed behaviors could be more prominent ${ }^{29}$. However, the stimulus-driven behaviors and the goal-directed behaviors were less prominent in schizophrenia ${ }^{30}$ and the equilibrium and the integration of two attention processes were deficient in psychopaths ${ }^{31,32}$. The similar consequence was also presented in the elders who performed more poorly than the younger in both attention processes, especially the goal-directed attention $^{33-35}$. By contrast, the goal-directed attention showed an age-equivalent modulation in the visual selective attention $^{36}$. Furthermore, aging goal-directed behaviors showed a weakened activation from occipitotemporal areas but increased contributions of anterior regions $s^{37,38}$.

To investigate directly and concretely those two attention processes, we used a visual search paradigm that combined the stimulus-driven attention with the goal-directed attention creatively ${ }^{26,39}$. In this paradigm, a target appeared among 3 distracters in separate stimulus-driven attention in which the distracters and the target were different in both color and orientation so that the target was seen primarily to draw attention automatically, and the goal-directed attention in which the distracters and the target were different only in orientation so that making more effort to find the target. Meanwhile, to better observe the changes of two attention processes under the HA environment, we would use ERPs as a measure owing to their preeminent temporal resolution and topography of ongoing neural activity of behaviors and their sensitivity to reveal processes that are not overt in behavior ${ }^{40}$. For those details that two attention processes evoked an early P1 and N1 component ${ }^{41-44}$ and the goal-directed attention generated a typically larger N1 component and more decreased P3 component than the stimulus-driven attention $^{21,26,45}$, we used the P1, N1 and P3 components to measure this task systematically that was practised on participants containing young inhabitants in the LA area and those who were born and grew up in sea level areas but then migrated to Tibet for a relatively long period. We hypothesized that two attention processes have poor performance in young immigrants in Tibet which can be indexed by changes of the ERP component.

\section{Results}

Behavioral Results. Table 1 illustrates the mean RTs and accuracy rates of each group. The results of a twoway ANOVA with condition (stimulus-driven attention and goal-directed attention) and group (LA group and HA group) as factors showed that the main effect of condition was significant for mean $\mathrm{RTs}[F(1,56)=838.83$; $\left.p<0.001 ; \eta_{p}^{2}=0.94\right]$, with slower RTs in the goal-directed attention than the stimulus-driven attention. In addition, the result of a one-way ANOVA demonstrated that there was a significant difference between groups in the stimulus-driven attention $[F(1,56)=4.40 ; p=0.04]$, slower RTs in the HA group than in the LA group. But no significant differences between groups were found in RTs for the goal-directed attention $(p>0.05)$. The two-way ANOVA showed that there was a main effect of condition for accuracy rates $[F(1,56)=58.07 ; p<0.001$; $\left.\eta_{p}^{2}=0.51\right]$, with more errors in the goal-directed attention than in the stimulus-driven attention. However, no significant differences were found in accuracy rates for either condition between two groups $(p>0.05)$.

ERP Results. P1. Figure 1B presents the mean change of ERPs at the PO7 and PO8 electrodes for the stimulus-driven attention and the goal-directed attention in either group, and Table 2 summarizes the P1 mean amplitude. A three-way ANOVA was performed with electrode location (PO7 and PO8), condition (stimulus-driven attention and goal-directed attention) and group (LA group and HA group) as factors. There was a main effect of group $\left[F(1,56)=9.84 ; p=0.003 ; \eta_{p}^{2}=0.15\right]$, with a smaller P1 amplitude in the HA group than in the LA group. Further the result of a one-way ANOVA found a smaller P1 amplitude in the HA group than in the LA group at PO7 electrode and PO8 electrode of the stimulus-driven attention [PO7: $F(1,56)=7.92$; $p=0.007 ; \eta_{p}^{2}=0.12$; PO8: $\left.F(1,56)=10.25 ; p=0.002 ; \eta_{p}^{2}=0.16\right]$ and the goal-directed attention [PO7: $F(1,56)=5.84 ; p=0.02 ; \eta_{p}^{2}=0.09 ;$ PO8: $\left.F(1,56)=7.20 ; p=0.01 ; \eta_{p}^{2}=0.11\right]$.

N1. The N1 mean waveforms in sensory-perceptual regions can be seen in Table 2 and Fig. $1 \mathrm{~B}$. In a three-way ANOVA with electrode location (PO7 and PO8), condition (stimulus-driven attention and goal-directed attention) and group (LA group and HA group) as factors, there was only a main effect of condition $[F(1,56)=11.33$; $\left.p=0.001 ; \eta_{p}^{2}=0.17\right]$, with greater amplitude in the goal-directed attention than in the stimulus-driven attention. Furthermore, the result of a one-way ANOVA that was performed with group (LA group and HA group) as factor for the goal-directed attention showed that there was a group difference at PO8 electrode $[F(1,56)=4.23 ; p=0.04$; $\left.\eta_{p}^{2}=0.07\right]$, with a smaller amplitude in the HA group than in the LA group. Further, a one-way ANOVA was performed with electrode location (PO7 and PO8) as factor in the stimulus-driven attention and the goal-directed attention for either group. Only in the LA group, N1 amplitude was greater at PO8 electrode than at PO7 

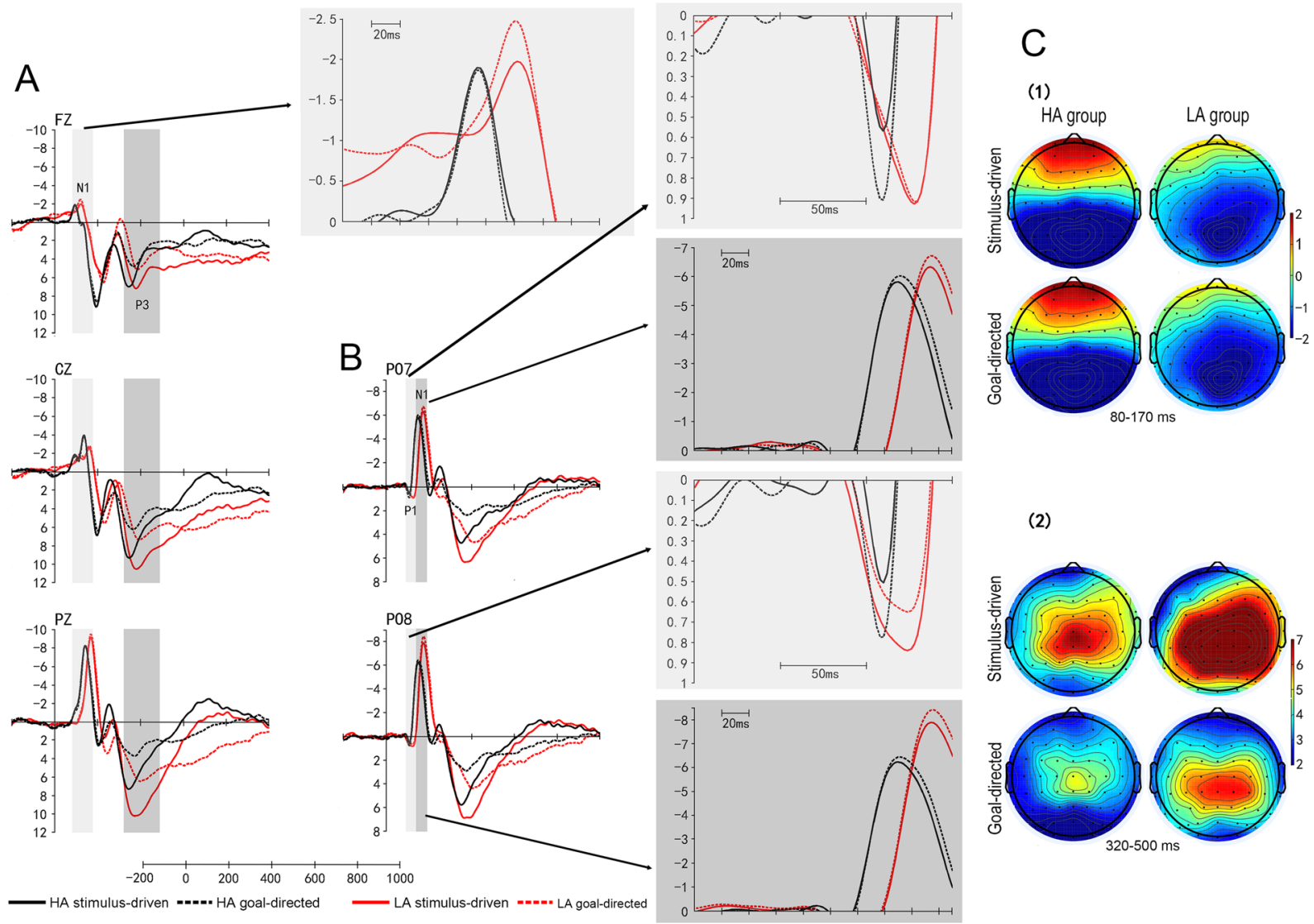

Figure 1. ERP waveforms and topographic map of two groups. (A) Averaged ERPs at FZ, CZ, and PZ electrodes for the stimulus-driven attention and the goal-directed attention in the HA group and the LA group. (B) Averaged ERPs at PO7 and PO8 electrodes for the stimulus-driven attention and goal-directed attention in the HA group and the LA group. (C) (1) Scalp topographies of the mean amplitude within the N1 time-window for the stimulus-driven attention (upper) and the goal-directed attention (bottom) attention in the HA group (left) and the LA group (right). (C) (2) Scalp topographies of the mean amplitude within the P3 time-window for the stimulus-driven attention (upper) and the goal-directed attention (bottom) attention in the HA group (left) and the LA group (right).

\begin{tabular}{|l|l|l|l|l|}
\hline & P1 amplitude & N1 amplitude \\
\cline { 2 - 5 } & HA group & LA group & HA group & LA group \\
\hline PO7 & $-0.92 \pm 2.10$ & $0.67 \pm 2.21$ & $-5.56 \pm 3.16$ & $-6.22 \pm 4.89$ \\
\hline Stimulus-driven & $-0.73 \pm 2.14$ & $0.65 \pm 2.21$ & $-5.79 \pm 3.07$ & $-6.59 \pm 4.60$ \\
\hline Goal-directed & \multicolumn{5}{|l|}{} \\
\hline PO8 & $-1.07 \pm 2.51$ & $0.70 \pm 1.60$ & $-6.00 \pm 3.76$ & $-7.62 \pm 4.64$ \\
\hline Stimulus-driven & $-0.91 \pm 2.45$ & $0.52 \pm 1.52$ & $-6.21 \pm 3.87$ & $-8.42 \pm 4.32$ \\
\hline Goal-directed
\end{tabular}

Table 2. P1, N1 mean amplitudes $(\mu \mathrm{V})$ and their corresponding standard deviations at PO7 and PO8 electrodes for each of two attention processes (Stimulus-driven attention; Goal-directed attention) in the HA and the LA groups.

electrode in the goal-directed attention $\left[F(1,28)=4.41 ; p=0.045 ; \eta_{p}^{2}=0.14\right]$. Figure $1 \mathrm{C}(1)$ further presents the scalp topographies of the mean amplitude within the N1 time window, showing that the hemisphere asymmetry was only found in LA group for two conditions, while the left and the right electrode sides were both activated in the HA group for two conditions.

Figure 1A shows the N1 amplitudes for the stimulus-driven attention and the goal-directed attention at FZ, $\mathrm{CZ}$, and PZ electrodes in either group. Table 3 presents the N1 mean amplitude. A three-way ANOVA with electrode location (FZ, CZ, and PZ), condition (stimulus-driven attention and goal-directed attention) and group (LA group and HA group) as factors demonstrated a main effect of electrode location (FZ, CZ, and PZ) 


\begin{tabular}{|l|l|l|l|l|l|l|}
\hline & FZ & CZ & PZ \\
\cline { 2 - 7 } & Stimulus-driven & Goal-directed & Stimulus-driven & Goal-directed & Stimulus-driven & Goal-directed \\
\hline N1 amplitude \\
\hline HA group & $0.83 \pm 2.74$ & $0.87 \pm 2.74$ & $-1.68 \pm 3.49$ & $-1.59 \pm 3.39$ & $-8.06 \pm 4.94$ & $-8.07 \pm 5.07$ \\
\hline LA group & $-0.71 \pm 2.12$ & $-0.96 \pm 1.94$ & $-1.54 \pm 2.59$ & $-1.76 \pm 2.64$ & $-8.59 \pm 5.87$ & $-8.88 \pm 6.02$ \\
\hline P3 amplitude \\
\hline HA group & $4.37 \pm 4.06$ & $3.30 \pm 3.12$ & $6.76 \pm 4.77$ & $4.91 \pm 4.03$ & $4.92 \pm 4.21$ & $2.74 \pm 3.88$ \\
\hline LA group & $5.51 \pm 4.63$ & $3.47 \pm 3.88$ & $8.91 \pm 5.55$ & $6.00 \pm 4.77$ & $8.67 \pm 5.58$ & $5.42 \pm 5.53$ \\
\hline
\end{tabular}

Table 3. $\mathrm{N} 1$ and $\mathrm{P} 3$ mean amplitudes $(\mu \mathrm{V})$ and their corresponding standard deviations at FZ, CZ, and PZ electrodes for each of two attention processes (Stimulus-driven attention; Goal-directed attention) in the HA and the LA groups.

\begin{tabular}{|c|c|c|c|c|}
\hline Variable & $t$ & $p$ & Beta & Model $R^{2}$ \\
\hline \multicolumn{5}{|l|}{ LA group } \\
\hline $\mathrm{FZ}$ & -2.48 & 0.02 & -0.31 & \multirow{3}{*}{0.10} \\
\hline $\mathrm{CZ}$ & -0.64 & 0.52 & -0.13 & \\
\hline $\mathrm{PZ}$ & -0.93 & 0.36 & -0.14 & \\
\hline \multicolumn{5}{|c|}{ HA group } \\
\hline FZ & -0.51 & 0.61 & -0.10 & \multirow{3}{*}{0.21} \\
\hline $\mathrm{CZ}$ & -3.86 & 0.00 & -0.46 & \\
\hline $\mathrm{PZ}$ & -0.72 & 0.48 & -0.13 & \\
\hline
\end{tabular}

Table 4. Stepwise regression of the averaged RTs (ms) using P3 amplitudes $(\mu \mathrm{V})$ at FZ, CZ, and PZ electrodes as predictors.

$\left[F(1,66)=153.52 ; p<0.001 ; \eta_{p}^{2}=0.73\right]$, with $\mathrm{PZ}>\mathrm{CZ}>\mathrm{FZ}$. Further, the results of a one-way ANOVA showed that there was a smaller amplitude in the HA group than in the LA group at FZ electrode for either condition [stimulus-driven attention: $F(1,56)=5.76 ; p=0.02 ; \eta_{p}^{2}=0.09$; goal-directed attention: $F(1,56)=8.65$; $\left.p=0.005 ; \eta_{p}^{2}=0.13\right]$.

P3. The amplitude of the P3 component is shown in Table 3 and Fig. 1A. The results of condition $\times$ electrode $\times$ group ANOVA suggested a significant main effect of condition and electrode [condition effect: $F(1,56)=62.37 ; p<0.001 ; \eta_{p}^{2}=0.53$; electrode effect: $F(1,80)=14.86 ; p<0.001 ; \eta_{p}^{2}=0.21$, with a greater amplitude in the stimulus-driven attention than in the goal-directed attention and $\mathrm{CZ}>\mathrm{PZ}>\mathrm{FZ}$. And there was a marginality difference between groups $\left[F(1,56)=3.14 ; p=0.08 ; \eta_{p}^{2}=0.05\right]$, with a smaller amplitude in the HA group than in the LA group. Furthermore, these was a significant interaction between condition and electrode $\left[F(1,78)=16.18 ; p<0.001 ; \eta_{p}^{2}=0.22\right]$ and between electrode and group $\left[F(1,80)=4.02 ; p=0.03 ; \eta_{p}^{2}=0.07\right]$. The results of the former showed that there was a greater amplitude for the stimulus-driven attention compared with the goal-directed attention at FZ, CZ and PZ electrodes, respectively. Meanwhile, the results showed $\mathrm{CZ}>\mathrm{PZ}>$ $\mathrm{FZ}$ in the stimulus-driven attention and $\mathrm{CZ}>\mathrm{FZ}, \mathrm{CZ}>\mathrm{PZ}$ in the goal-directed attention. And the results of the later showed a greater amplitude at $\mathrm{CZ}$ and $\mathrm{PZ}$ electrodes than at FZ electrode in the LA group and a greater amplitude at CZ electrode than at FZ and PZ electrodes in the HA group. Meanwhile, there was a smaller amplitude in the HA group than in the LA group at PZ electrode $(p=0.01)$. Further, the results of a one-way ANOVA showed that there was a smaller amplitude in the HA group than in the LA group at PZ electrode for either condition [stimulus-driven attention: $F(1,56)=8.36, p=0.005 ; \eta_{p}^{2}=0.13$; goal-directed attention: $F(1,56)=4.56$; $\left.p=0.04 ; \eta_{p}^{2}=0.08\right]$. Figure $1 \mathrm{C}(2)$ shows the scalp topographies of the mean amplitude within the $\mathrm{P} 3$ time-window for either group, which are similar to the results of the anterior three-way ANOVA about P3 amplitude for either group. In the left column of Fig. $1 \mathrm{C}(2)$, a larger activity appeared at central region for the stimulus-driven attention and at fronto-central region for the goal-directed attention in the HA group, while right column of Fig. 1C(2) generates the tendency of the central-parietal distribution for the stimulus-driven attention and the tendency of the central distribution for the goal-directed attention in the LA group.

Correlation analyses. Table 4 shows a stepwise regression of RTs and P3 amplitude between two groups. The first model only included the data of the LA group, the final significant predictor was FZ that was related negatively to RTs and accounted for $10 \%$ of the variance. The second model only included the data of the HA group, the final significant predictor was CZ that was related negatively to RTs and accounted for $21 \%$ of the variance.

\section{Discussion}

The departure point for this study was to explore the performance of the two attentional processes following impaired visual attention behavior in healthy young immigrants under the HA environment in Tibet. We found the difference of RTs in the stimulus-driven attention between groups, longer RTs in the HA group. On this basis, we further discovered cognitive resources of the stimulus-driven attention and goal-directed attention 
behaviors were insufficient in the HA group and the goal-directed attention had an activation of the anterior region at the late stage in the HA group. Moreover, behavior response and resources allocation exhibited a negative correlation at central region in the HA group. These results provided experimental evidence for the changes of stimulus-driven and goal-directed attention behaviors as a result of the HA exposure.

The behavioral results, in a consistence with previous studies ${ }^{26,28}$, showed that there was a longer RTs and higher error rates in the goal-directed attention than in the stimulus-driven attention, suggesting that the paradigm indeed had distinguished two attention processes. Moreover, the HA group had a longer RTs compared to the LA group only in the stimulus-driven attention, showing the slower processing of the stimulus-driven attention in the HA group.

In this study, we first found that the P1 component showed a great difference between two groups, a smaller posterior P1 amplitude in the HA group compared to the LA group for either attention process. Meanwhile, there was a smaller right posterior N1 only for the goal-directed attention in the HA group than in the LA group. Some studies have indicated that several universal ERP components of the attention contain the parietal-occipital P1 and $\mathrm{N} 1^{46-48}$ that are indexes of the attentional allocation of the early visual processing involved in alerting and orienting and are enhanced under conditions of the difficult attention tasks ${ }^{47,49-52}$. In our study, this difference between two groups for the P1 component might imply that the early alerting and orienting processing of two attention processes depressed in the HA group. The difference of right posterior N1 only for the goal-directed attention between two groups suggested the subdued expression of the goal-directed attention in the early visual processing. The convergent result of above consequences was presented in the distribution difference of N1 component again. There was a hemispheric difference of the $\mathrm{N} 1$ amplitude for the goal-directed attention only in the LA group, with a larger $\mathrm{N} 1$ amplitude at the right hemisphere than at the left hemisphere, while there was no difference between the two hemispheres for the goal-directed attention in the HA group. Besides, the distribution of N1 amplitude (Fig. 1C(1)) also showed that only the right posterior sites were active for two attention processes in the LA group, while bilateral posterior sites were active in the HA group for two attention processes. Remarkably, the parietal-occipital N1 component usually shows hemispheric asymmetry, especially displaying the dominance of the right hemisphere in visual-spatial attention tasks $s^{53-57}$. This dominance of the right hemisphere about the N1 amplitude only in the LA group was similar to previous studies demonstrating the altitude effect on the dominance of the right hemisphere ${ }^{2}$. And this bilateral activation in the HA group also verified the altitude effect on compensatory mechanism for the left hemisphere in attention again ${ }^{2}$ suggesting that the HA group had to invoke bilateral resources to input more effort for two attention processes due to the lack of mental resources. In frontoparietal networks, there was a parallel result between groups, a smaller N1 amplitude in the HA group than in the LA group at the frontal area for either attention process. Note that the frontal $\mathrm{N} 1$ components are larger for the attended locations compared with those unattended ${ }^{58}$ in line with the sensory gain control mechanism in which the amount of attention distribution determines the magnitude of the evoked neural activity ${ }^{59}$. So the difference on the frontal N1 between groups also indicates the insufficient amount of attention allocation to the two attention processes in the HA group compared with the LA group.

This performance of decreased mental resources to two attention processes was also reflected in the later P3 component, whose scalp distributions typically located at parietal region ${ }^{60}$. The P3 component is regarded as an index of the allocation of attentional resources in the later conscious processing ${ }^{37,61-63}$ that involves the evaluation processes of stimulus meaning ${ }^{49,61}$. The present result showed a greater P3 amplitude in the stimulus-driven attention than in the goal-directed attention according with previous studies which discovered that the evaluation of novel or salient stimuli, such as the bottom-up condition ${ }^{26}$, pop-out arrays ${ }^{64,65}$ and negative pictures ${ }^{66}$, evoked a larger P3 amplitude because these stimuli had greater information value to attract more interests in survival surroundings. This result therefore suggested that the shorter RTs observed in the stimulus-driven attention than the goal-directed attention might owe much to more allocation of resources to evaluate the stimuli. Compared to the LA group, there was a smaller amplitude in the HA group at the parietal area for either attention process. This result made clear that the HA group might have insufficient resources sequentially so that put less into the evaluation of information meaning for the two attention processes, in accord with previous normal aging studies finding that the parietal P3 amplitude decrease with age $\mathrm{e}^{37,67,68}$.

These results of P1, N1 and P3 component suggested that in the two attention processes, young immigrants with long-term high altitude exposure performed more poorly than young inhabitants in the LA area, which was consistent with the age-related decrease of the two attention processes ${ }^{26,34,35}$. In addition, the two subprocedures of attention had different performance on the P3 scalp distribution between two groups, as shown in Fig. 1C(2), a central-parietal P3 for the stimulus-driven attention in the LA group, whereas a maximum central P3 for the stimulus-driven attention in the HA group. Previous studies have consistently reported that a parietal maximum P3 component amplitude appears in easy tasks, whereas central maximum P3 component amplitude appears in difficult tasks ${ }^{69}$. These implied that the stimulus-driven attention was difficult for the HA group because of the shortage of mental resources. For the goal-directed attention, there was a central tendency P3 in the LA group, whereas a fronto-central P3 in the HA group, suggesting a more anterior activation distribution and a underactivation of parieto-occipital region in the HA group. These results were in concordance with the aging findings that frontal regions were more involved in the goal-directed attention than posterior, sensory regions, in aging ${ }^{26}$. Universally, some studies observed a underactivation due to brain regions damage in aging ${ }^{70-72}$ that suggested frequently worse performance in the memory, cognitive control and executive control behaviors ${ }^{73}$. Reversely, an unexpected pattern started to appear which showed the activation of brain regions in the older adults, Alzheimer's disease patients and the mild cognitive impairment patients ${ }^{73}$, especially the frontal area related with the executive control of attention to the environment, suggesting different strategies to compensate for poorer effects of brain damage in higher-level processing ${ }^{73,74}$. Further, a region worked harder resulting in activation due to declining input it received, indicating a compensation for descending processing elsewhere ${ }^{73,74}$. In some studies, more greater prefrontal activity had an adjoint underactivation of occipitotemporal areas in the elder, supporting the 
view that strategic processes mediated by the prefrontal regions compensated for inefficient sensory processing in the visual pathway with age to reach age-equivalent performance ${ }^{75-78}$. It was important to note that the present study found homologous result in the goal-directed attention of the HA group and there was a smaller posterior $\mathrm{N} 1$ and P3 in the HA group than the LA group. By above account, we speculated that the HA group industriously activated more anterior region to compensate for the declining efficiency of sensory processing regions so that the HA group could perform successfully the task of the goal-directed attention and had equivalent performance compared with the LA group, as evidenced by non-significant RTs in the goal-directed attention between two groups. This conformed with other aging studies of attention that activation of anterior region reflected an improved performance in the elders ${ }^{26,79,80}$. Meanwhile, those results declared the separation of two attention processes under deficient resources due to HA environment, a compensation to insufficient resources of posterior area only in the goal-directed attention although insufficient resources in two attention processes, that might state people was more inclined to explore expression of internal volition under HA environment, such as meditation behavior.

Our findings were consistent with Kahneman's limited-capacity model of attention ${ }^{81}$. It was believed that the total amount of attention resources is limited. But if the sum of resources that the two tasks required is no more than the total number of psychological resources allocated at the same time, it is possible to operate both tasks at the same time. If not, two tasks would interfere with each other, thereby affected effective execution of both tasks. We thought that cognitive resources were insufficient in the HA group similar to the normal aging ${ }^{37}$, so that the two attention processes might be out of control, which mainly manifested as the impairment of two attention processes and compensation for only goal-directed attention. Further, we found that there were certain relationships that supported by evidence from the results of stepwise regression between the amount of resources allocation that this limitation generated and the performance of attention behaviors. The results showed that this correlation was negative between P3 amplitude and RTs in both groups, indicating that the decreasing P3 amplitude was associated with longer RTs, that is, the diminishing resource was associated with the slower responses. In addition, the results of within-group stepwise regression manifested that the relationship between the $\mathrm{P} 3$ amplitude and RTs had a different performance between two groups. Significant predictor was FZ in the LA group and significant predictor was CZ in the HA group, indicating that the LA group with diminishing resource of the frontal area exhibited slower responses and the HA group with diminishing resource of the central area exhibited slower responses.

There were some considerations to interpret the present findings. First, although the difference on ERP components indicated the neural mechanism of the declining attention behavior had some changes under long-term HA exposure, the related results should still be interpreted cautiously, because they may be influenced by some social factors (e.g. economic status, education level or culture) ${ }^{82}$. However, we suggest that hypoxia is leading cause of observed changes as this is most important factor of the HA environment. Although the brain accounts for only about $2 \%$ of the body's weight, the brain's oxygen consumption is up to $20 \%$ of the total ${ }^{83-85}$. Therefore, the central nervous system is particularly sensitive to hypoxia, which can cause changes in the brain function and neurons ${ }^{86}$. Previous researches in which inhabitants in the LA area accepted the acute exposure to hypoxia or simulated hypoxia conditions or moderate hypoxia (under the condition of 8 hours hypoxia) found that hypoxia has an effect on attention behavior progressing ${ }^{10,87}$ and executive function ${ }^{88}$. Obviously, hypoxia affect the behavior most directly and most quickly. So we need to find a way to further prove it in the future, although it is not easy. Second, the expression of goal-directed attention exposed to the HA environment need to be considered. Theory of environmental stress believed that various factors in the environment could cause people's stress response and the view of molecular medicine also declared that the brain tissue created stress response when people exposed to a deleterious environment ${ }^{89}$. Via a coordinated stress response, the brain could avoid or decrease neurologic damage ${ }^{90}$. This indicated that the brain had the potential capacity of self-protection, such as endogenous neuroprotection, in which the brain could confront these threats of hypoxia or hypothermia ${ }^{91,92}$. Connecting with the fact that the goal-directed activity was main aspect of brain function ${ }^{84}$, we speculated that our finding about the preemptive compensation on the goal-directed attention might reflect a mechanism of cerebral protection under the HA environment. Third, these results did not indicate the essence of the present observation reflecting the deficit of the attention function or the adaption of the attention function in the high altitude exposure which is what we need do in the future. Fourth, because attention ability was a very important part of cognitive function, the present study that focused on the altitude effect on attention behavior reflected broadwise the changes of cognitive function, but it must be admitted that the HA environment might affect all sorts of aspects of cognitive function. So the follow-up studies need further investigate the interrelation about various aspects of cognitive function including perception, memory, decision-making and so on. Finally, the cross-sectional nature of the study also is a limitation. Note that this issue about effects of HA environment exposure on brain function is just getting started, so we have used the cross-sectional study to explore this issue at this stage. Certainly, the dynamic longitudinal study is an important research direction and we will do it in the future.

In conclusion, the present study found different expression of two attention behaviors under the HA environment in Tibet versus LA environment at sea level. The cognitive resources of entire attention behaviors were inadequate and this magnitude of resources allocation and behavior response were negatively correlated. The stimulus-driven behaviors and the goal-directed behaviors performed more poorly and the goal-directed behaviors had a compensation of anterior region for descending efficiency of sensory processing as a result of the HA exposure at later stage.

\section{Methods}

Participants. A total of 58 healthy, right-handed college students (18-25 years old) were recruited for the present study. All of these participants belong to Han race, in which 29 students from Tibet University were defined as HA group, and the rest from colleges and universities in Guangzhou were categorized into LA group. The participants of the HA group had lived in HA $(3,650 \mathrm{~m})$ for 3 years and more, but were born and grew up in 


\begin{tabular}{|c|c|c|c|}
\hline & & $\mathrm{M} \pm \mathrm{SD}$ & $p$ \\
\hline \multirow{2}{*}{ CEE } & HA & $505.41 \pm 24.98$ & \multirow{2}{*}{0.26} \\
\hline & LA & $521.86 \pm 74.51$ & \\
\hline \multirow{2}{*}{ RIT } & HA & $46.45 \pm 9.26$ & \multirow{2}{*}{0.13} \\
\hline & LA & $50.14 \pm 8.87$ & \\
\hline \multirow{2}{*}{ PSQI } & HA & $5.32 \pm 2.65$ & \multirow{2}{*}{0.82} \\
\hline & LA & $5.15 \pm 2.64$ & \\
\hline \multirow{2}{*}{ Time } & HA & $4.07 \pm 0.70$ & \multirow{2}{*}{.000} \\
\hline & LA & $0.00 \pm 0.00$ & \\
\hline
\end{tabular}

Table 5. The demographic data of the HA and LA groups. Note: CEE, the scores on the college entrance Examination; RIT, the scores of the Raven Intelligence Test; PSQI, the scores of sleep; Time, the time of staying at the plateau.

LA areas $(<1,000 \mathrm{~m})$. The participants of the LA group had lived in LA areas all the time. The Raven Intelligence Test $^{93}$ and basic information questionnaire including the scores on the college entrance examination, sleep, and the time of staying at the plateau were measured before the experiment, and there was no significant difference between the two groups in their scores on the Raven Intelligence Test $(p>0.05)$, minimizing the likelihood that any group differences could be explained by intelligence-related factors (see Table 5). All the participants had normal or corrected-to-normal vision with no prior neurological problems. None of them were taking any psychotropic, neurological, or psychiatric medications at the time of testing. The informed consent was obtained for each participant, and the experiment was commanded in accordance with the Declaration of Helsinki and was approved by the Ethics Committee of the Institute of Psychology, South China Normal University.

Stimuli and procedure. The visual stimuli were the 16 particular triangles which had different colors (red or green) and orientations $\left(45^{\circ}, 90^{\circ}, 135^{\circ}, 180^{\circ}, 225^{\circ}, 270^{\circ}, 315^{\circ}\right.$, and $360^{\circ}$ from the vertical meridian). They were combined into "stimulus-driven attention" and "goal-directed attention" conditions which were consisted of 4 triangles respectively whose positions were distributed in the 4 quadrants resulting in stimuli subtending a total $5.34^{\circ}$ of visual angle from the fixation cross and which consisted of the target and 3 disturbed items. In the "stimulus-driven attention" condition, 3 disturbed triangles were differed from the target in color and orientation, which generated target stimulus to be so salient that could grab it easily. In the "goal-directed attention" condition, 3 disturbed triangles were differed from the target only in orientation, which caused the target stimulus to be so imperceptible in the scene that should make a special effort to search it. All conditions were randomized and equal, and the targets appeared randomly in the left visual field or right visual field.

Figure 2 illustrates an example of the stimulus sequence. A trial begin with a $500 \mathrm{~ms}$ fixation cross, followed by a target triangle which had a specific color and orientation and was presented for $1000 \mathrm{~ms}$. Then $500 \mathrm{~ms}$ delay screen with a fixation cross which maintained subjects' central fixation, and followed by the "stimulus-driven attention" or the "goal-directed attention". Participants were asked to press "F" when targets appeared on the left side of the screen or "J" when targets were on the right side as quickly and accurately as possible, regardless of the vertical position. If no response was made, the array remained on the screen until the participant responded, followed by a $1000 \mathrm{~ms}$ green fixation cross to signal the end of the trial. Participants sat in a dimly-lit and quiet room to perform this visual search paradigm which was presented against a black background on a 19-inch computer monitor at a distance of $110 \mathrm{~cm}$. They should complete 12 blocks and each block had 32 trials, resulting in 192 trials in each condition. Sufficient practice was provided to make sure that all participants' accuracy rate could reach more than $80 \%$ before starting the experiment. Extra practice blocks were given as required until participants were able to reach a mean accuracy of $80 \%$ in the task. After each block, participants could have a rest.

The stimuli were presented and the behavioral data were collected and analyzed via $E$-Prime software (Version 2.0, Psychology Software Tools, Pittsburgh, PA).

ERP Recordings and Data Analysis. Brain electrical activity was recorded via 64-channel electrode cap (10/20 system) using Ag/AgCl electrodes in Neuroscan Curry software (version 7.8), with an on-line reference to the middle of the CZ and CPZ locations. Simultaneously, six additional electrodes were recorded including M1 and M2 (because off-line re-reference was the average of the left and right mastoid) and four electrooculogram (EOG). EOG were recorded by placing one electrode above and below the left eye to measure vertical electrooculogram (VEOG) and the outer canthi of both eyes to record horizontal electrooculogram (HEOG), respectively. Ultimately, EEG and EOG were continuously recorded at a sampling rate of $500 \mathrm{~Hz}$, applying less than $5 \mathrm{k} \Omega$ electrode impedances.

Trials contaminated by body movements, eye movements, and muscle activity, were rejected offline, with a criterion of $\pm 100 \mu \mathrm{V}$. Only trials with correct responses were averaged and segmented into stimulus-locked ERP segments, from $200 \mathrm{~ms}$ (baseline) prior to the appearance of stimuli to $1000 \mathrm{~ms}$ afterwards, in which the ERP was digitally filtered with a $0.1 \mathrm{~Hz}-30 \mathrm{~Hz}$ band pass filter. Following these analyses, each participant averagely retained about 163 trials for the stimulus-driven attention and 162 trials for the goal-directed attention in the LA group, and retained about 162 trials for the stimulus-driven attention and 161 trials for the goal-directed attention in the HA group. 


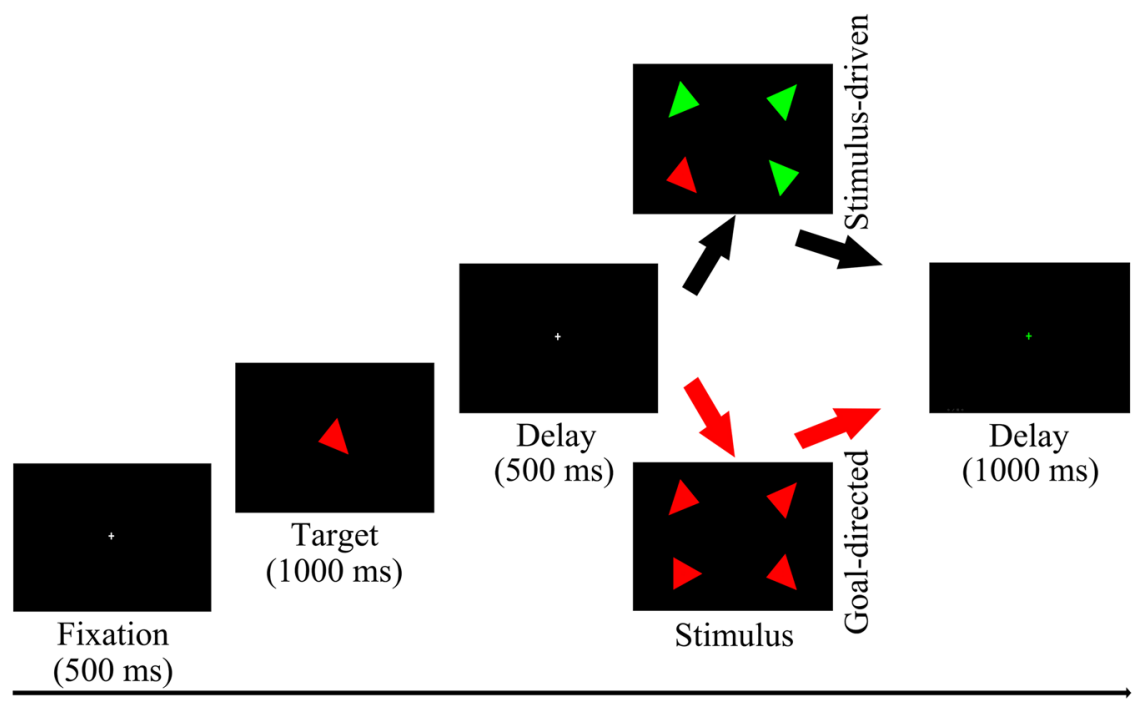

Figure 2. Stimulus sequence of the visual search paradigm.

In stimulus-locked ERP segments, experimental condition elicited clear P1, N1 and P3 components in either group. To reduce the effect of noise, we measured the mean voltage of every component over a time window in which we could find a peak amplitude according to Luck's recommendation ${ }^{40}$. To observe the early perceptual processing in the stimulus-driven attention and the goal-directed attention for either group, P1 component and $\mathrm{N} 1$ component were measured at the PO7 and PO8 electrodes in the $100 \mathrm{~ms}-135 \mathrm{~ms}$ and $140 \mathrm{~ms}-185 \mathrm{~ms}$ time window, respectively. In addition, considering close connections between the frontoparietal networks and two attention processes ${ }^{94-97}$, three electrodes (FZ, CZ, and PZ) were selected for N1 and P3 components data analysis in the $80 \mathrm{~ms}-170 \mathrm{~ms}$ and $320 \mathrm{~ms}-500 \mathrm{~ms}$ time windows, respectively, to compare the changes of frontoparietal processing in the stimulus-driven attention and the goal-directed attention for either group.

All data were analyzed with SPSS (SPSS, Inc., Chicago). ANOVA was performed on mean RTs for correct responses, accuracy rates and ERP data, whose statistically significance level was set at 0.05 and degrees of freedom of the $F$ ratios were corrected according to the Greenhouse-Geisser method. Besides, in order to understand the relation between behavioral expression and resources allocation, a stepwise regression analysis was performed to explore the relationship between dependent variable RTs and predictor variables P3 mean amplitudes at FZ, $\mathrm{CZ}$, and PZ electrodes.

\section{References}

1. Viruésortega, J., Garrido, E., Javierre, C. \& Kloezeman, K. C. Human behaviour and development under high-altitude conditions. Developmental Science 9, 400-410 (2006).

2. Wang, Y. et al. Long-Term Exposure to High Altitude Affects Voluntary Spatial Attention at Early and Late Processing Stages. Scientific Reports 4, 1-8 (2014).

3. Yan, X. Cognitive impairments at high altitudes and adaptation. High Alt Med Biol 15, 141-145, https://doi.org/10.1089/ ham.2014.1009 (2014).

4. Martin, D. S., Levett, D. Z. H., Grocott, M. P. W. \& Montgomery, H. E. Variation in human performance in the hypoxic mountain environment. Experimental Physiology 95, 463-470, https://doi.org/10.1113/expphysiol.2009.047589 (2010).

5. Corbetta, M. \& Shulman, G. L. Control of goal-directed and stimulus-driven attention in the brain. Nature reviews. Neuroscience 3 , 201-215 (2002).

6. Cave, K. R. \& Wolfe, J. M. Modeling the role of parallel processing in visual search. Cognitive Psychology 22, 225-271, https://doi. org/10.1016/0010-0285(90)90017-X (1990).

7. Desimone, R. \& Duncan, J. Neural mechanisms of selective visual attention. Annu Rev Neurosci 18, 193-222, https://doi.org/10.1146/ annurev.ne.18.030195.001205 (1995).

8. Kastner, S. \& Ungerleider, L. G. Mechanisms of visual attention in the human cortex. Annu Rev Neurosci 23, 315-341, https://doi. org/10.1146/annurev.neuro.23.1.315 (2000)

9. Yilmaz, O. Mechanisms of spatial localization in the human visual system. Dissertations \& Theses - Gradworks (2007).

10. Stivalet, P. et al. Positive expiratory pressure as a method for preventing the impairment of attentional processes by hypoxia. Ergonomics 43, 474-485 (2000).

11. Thakur, L., Ray, K., Anand, J. \& Panjwani, U. Event related potential (ERP) P300 after 6 months residence at 4115 meter. The Indian journal of medical research 134, 113 (2011).

12. Penaloza, D. \& Ariasstella, J. The heart and pulmonary circulation at high altitudes: healthy highlanders and chronic mountain sickness. Circulation 115, 1132-1146 (2007)

13. Liao, S. B. Quantitative Analysis of Relationship Between Population Distribution and Environmental Factors in Qinghai-Tibet Plateau. China Population Resources \& Environment (2003).

14. Wu, T. \& Kayser, B. High Altitude Adaptation in Tibetans. High Altitude Medicine \& Biology 7, 193-208 (2006).

15. Bonnon, M., Noel-Jorand, M. \& Therme, P. Effects of different stay durations on attentional performance during two mountain expeditions. Aviation, space, and environmental medicine 71, 678-684 (2000).

16. Chiu, G., Chatterjee, D., Johnson, R. \& Freund, G. The impact of acute hypoxia on learning and memory. Brain, Behavior, and Immunity 24, S40 (2010).

17. Wesensten, N. J. et al. Effects of simulated high altitude exposure on long-latency event-related brain potentials and performance. Aviat Space Environ Med 64, 30-36 (1993). 
18. Mort, D. J. et al. Differential cortical activation during voluntary and reflexive saccades in man. Neuroimage 18, 231-246 (2003).

19. Mayer, A. R., Dorflinger, J. M., Rao, S. M. \& Seidenberg, M. Neural networks underlying endogenous and exogenous visual-spatial orienting. Neuroimage 23, 534-541 (2004)

20. Berger, A., Henik, A. \& Rafal, R. Competition between endogenous and exogenous orienting of visual attention. Journal of Experimental Psychology-General 134, 207-220 (2005).

21. Hopfinger, J. B. \& West, V. M. Interactions between endogenous and exogenous attention on cortical visual processing. NeuroImage 31, 774-789 (2006).

22. Corbetta, M., Kincade, J. M., Ollinger, J. M., McAvoy, M. P. \& Shulman, G. L. Voluntary orienting is dissociated from target detection in human posterior parietal cortex. Nature neuroscience 3, 292 (2000).

23. Corbetta, M., Patel, G. \& Shulman, G. L. The reorienting system of the human brain: from environment to theory of mind. Neuron 58, 306-324 (2008)

24. Li, X. Attention and eye movement control: Interaction of top-down and bottom-up information. University of Massachusetts Amherst, (2007).

25. Itti, L. \& Koch, C. A saliency-based search mechanism for overt and covert shifts of visual attention. Vision Research 40, 1489-1506 (2000).

26. Li, L., Gratton, C., Fabiani, M. \& Knight, R. T. Age-related frontoparietal changes during the control of bottom-up and top-down attention: an ERP study. Neurobiology of aging 34, 477-488 (2013).

27. Li, L., Gratton, C., Yao, D. \& Knight, R. T. Role of frontal and parietal cortices in the control of bottom-up and top-down attention in humans. Brain research 1344, 173-184 (2010).

28. Madden, D. J. et al. Age mediation of frontoparietal activation during visual feature search. NeuroImage 102, $262-274$ (2014).

29. Yantis, S. \& Jonides. Abrupt visual onsets and selective attention: Voluntary versus automatic allocation. Journal of Experimental Psychology: Human Perception \& Performance. 16, 121-134 (1990).

30. Neuhaus, A. H. et al. Dissection of early bottom-up and top-down deficits during visual attention in schizophrenia. Clinical Neurophysiology 122, 90-98 (2011).

31. Newman, J. P. et al. Deficient integration of top-down and bottom-up influences on attention in psychopaths: Potential contribution of the septo-hippocampal system. Psych.wisc.edu (2006).

32. Dima, D., Dietrich, D. E., Dillo, W. \& Emrich, H. M. Impaired top-down processes in schizophrenia: a DCM study of ERPs. Neuroimage 52, 824-832 (2010).

33. Kok, A. Age-related changes in involuntary and voluntary attention as reflected in components of the event-related potential (ERP). Biological psychology 54, 107-143 (2000).

34. Lien, M.-C., Gemperle, A. \& Ruthruff, E. Aging and involuntary attention capture: electrophysiological evidence for preserved attentional control with advanced age. Psychology and aging 26, 188 (2011).

35. Madden, D. J., Whiting, W. L. \& Huettel, S. A. Age-related changes in neural activity during visual perception and attention. Cognitive neuroscience of aging: Linking cognitive and cerebral aging, 157-185 (2005).

36. Guerreiro, M. J., Anguera, J. A., Mishra, J., Van Gerven, P. W. \& Gazzaley, A. Age-equivalent top-down modulation during crossmodal selective attention. Journal of Cognitive Neuroscience 26, 2827-2839 (2014).

37. Lorenzolópez, L., Amenedo, E., Pascualmarqui, R. D. \& Cadaveira, F. Neural correlates of age-related visual search decline: a combined ERP and sLORETA study. Neuroimage 41, 511-524 (2008).

38. Friedman, D. Oxford Handbook of Event-Related Potential Components. Oxford Library of Psychology (2008).

39. Buschman, T. J. \& Miller, E. K. Top-down versus bottom-up control of attention in the prefrontal and posterior parietal cortices. science 315, 1860-1862 (2007).

40. Luck, S. J. Luck, S. J. (In press). Event-related potentials. In D. L. Long (Ed.), APA Handbook of Research Methods in Psychology. Washington, DC: American Psychological Association (2012).

41. Di Russo, F., Martínez, A. \& Hillyard, S. A. Source analysis of event-related cortical activity during visuo-spatial attention. Cerebral cortex 13, 486-499 (2003).

42. Heinze, H. et al. Combined spatial and temporal imaging of brain activity during visual selective attention in humans. Nature 372, 543 (1994).

43. Mangun, G. R., Hopfinger, J. B., Kussmaul, C. L., Fletcher, E. M. \& Heinze, H.-J. Covariations in ERP and PET measures of spatial selective attention in human extrastriate visual cortex. Human brain mapping 5, 273-279 (1997).

44. Martinez, A. et al. Involvement of striate and extrastriate visual cortical areas in spatial attention. Nature neuroscience 2 (1999).

45. Eason, R. G., Harter, M. R. \& White, C. Effects of attention and arousal on visually evoked cortical potentials and reaction time in man. Physiology \& Behavior 4, 283-289 (1969).

46. Di Russo, F., Martínez, A., Sereno, M. I., Pitzalis, S. \& Hillyard, S. A. Cortical sources of the early components of the visual evoked potential. Human brain mapping 15, 95-111 (2002).

47. Hillyard, S. A. \& Anllo-Vento, L. Event-related brain potentials in the study of visual selective attention. Proceedings of the National Academy of Sciences 95, 781-787 (1998).

48. Luck, S. J. \& Kappenman, E. S. The Oxford handbook of event-related potential components. Oxford university press (2011).

49. Campanella, S. et al. Discrimination of emotional facial expressions in a visual oddball task: an ERP study. Biological psychology 59, 171-186 (2002).

50. Barthélémy, S. \& Boulinguez, P. Orienting visuospatial attention generates manual reaction time asymmetries in target detection and pointing. Behavioural brain research 133, 109-116 (2002).

51. Talsma, D., Slagter, H. A., Nieuwenhuis, S., Hage, J. \& Kok, A. The orienting of visuospatial attention: An event-related brain potential study. Cognitive Brain Research 25, 117-129 (2005).

52. Williams, R. S. et al. Age differences in the Attention Network Test: Evidence from behavior and event-related potentials. Brain \& Cognition 102, 65 (2016).

53. Hsiao, F.-J., Hsieh, J.-C., Lin, Y.-Y. \& Chang, Y. The effects of face spatial frequencies on cortical processing revealed by magnetoencephalography. Neuroscience Letters 380, 54-59 (2005).

54. Haxby, J. V., Hoffman, E. A. \& Gobbini, M. I. The distributed human neural system for face perception. Trends in cognitive sciences 4, 223-233 (2000).

55. Puce, A., Allison, T., Asgari, M., Gore, J. C. \& McCarthy, G. Differential sensitivity of human visual cortex to faces, letterstrings, and textures: a functional magnetic resonance imaging study. Journal of neuroscience 16, 5205-5215 (1996).

56. Rossion, B., Joyce, C. A., Cottrell, G. W. \& Tarr, M. J. Early lateralization and orientation tuning for face, word, and object processing in the visual cortex. Neuroimage 20, 1609-1624 (2003).

57. Pinsk, M. A., DeSimone, K., Moore, T., Gross, C. G. \& Kastner, S. Representations of faces and body parts in macaque temporal cortex: a functional MRI study. Proceedings of the National Academy of Sciences of the United States of America 102, 6996-7001 (2005).

58. Vogel, E. K. \& Luck, S. J. The visual N1 component as an index of a discrimination process. Psychophysiology 37, 190-203 (2000).

59. SA, H., EK, V. \& SJ, L. Sensory gain control (amplification) as a mechanism of selective attention: electrophysiological and neuroimaging evidence. Philosophical transactions of the Royal Society of London. Series B, Biological sciences 353, 1257-1270 (1998).

60. Polich, J. Updating P300: an integrative theory of P3a and P3b. Clinical neurophysiology 118, 2128-2148 (2007). 
61. Campanella, S. et al. Human gender differences in an emotional visual oddball task: an event-related potentials study. Neuroscience letters 367, 14-18 (2004).

62. Goldstein, A., Spencer, K. M. \& Donchin, E. The influence of stimulus deviance and novelty on the P300 and novelty P3. Psychophysiology 39, 781-790 (2002).

63. Polich, J. \& Kok, A. Cognitive and biological determinants of P300: an integrative review. Biological Psychology 41, 103-146 (1995).

64. Luck, S. J. \& Hillyard, S. A. Electrophysiological evidence for parallel and serial processing during visual search. Attention, Perception, \& Psychophysics 48, 603-617 (1990).

65. Luck, S. J. \& Hillyard, S. A. Electrophysiological correlates of feature analysis during visual search. Psychophysiology 31, 291-308 (1994).

66. Ito, T. A., Larsen, J. T., Smith, N. K. \& Cacioppo, J. T. Negative information weighs more heavily on the brain: the negativity bias in evaluative categorizations. Journal of Personality \& Social Psychology 75, 887-900 (1998).

67. Fabiani, M. \& Friedman, D. Changes in brain activity patterns in aging: the novelty oddball. Psychophysiology 32, 579-594 (1995).

68. Friedman, D. The components of aging. Oxford handbook of event-related potential components, 513-536 (2012).

69. Polich, J. \& Comerchero, M. D. P3a from visual stimuli: typicality, task, and topography. Brain topography 15, 141-152 (2003).

70. Grady, C. et al. Age-related reductions in human recognition memory due to impaired encoding. Science (New York, N.Y.) 269, 218-221 (1995)

71. Cabeza, R., Anderson, N. D., Locantore, J. K. \& Mcintosh, A. R. Aging gracefully: compensatory brain activity in high-performing older adults. Neuroimage 17, 1394-1402 (2002).

72. Reuter-Lorenz, P. New visions of the aging mind and brain. Trends in Cognitive Sciences 6, 394-400 (2002)

73. Reuter-Lorenz, P. A. \& Lustig, C. Brain aging: reorganizing discoveries about the aging mind. Current Opinion in Neurobiology 15, 245 (2005).

74. Reuter-Lorenz, P. A. \& Cappell, K. A. Neurocognitive Aging and the Compensation Hypothesis. Current Directions in Psychological Science 17, 177-182 (2010).

75. Gutchess, A. H. et al. Aging and the Neural Correlates of Successful Picture Encoding: Frontal Activations Compensate for Decreased Medial-Temporal Activity. Journal of Cognitive Neuroscience 17, 84-96 (2014).

76. Buckner, R. L., Snyder, A. Z., Sanders, A. L. \& Raichle, M. E. Functional Brain Imaging of Young, Nondemented, and Demented Older Adults. Journal of Cognitive Neuroscience 12, 24-34 (2000).

77. Cabeza, R. \& Nyberg, L. Imaging cognition II: An empirical review of 275 PET and fMRI studies. Journal of Cognitive Neuroscience 12, 1-47 (2000).

78. Cabeza, R. et al. Task-independent and task-specific age effects on brain activity during working memory, visual attention and episodic retrieval. Cerebral Cortex 14, 364 (2004).

79. Madden, D. J., Whiting, W. L., Provenzale, J. M. \& Huettel, S. A. Age-related changes in neural activity during visual target detection measured by fMRI. Cerebral Cortex 14, 143-155 (2004).

80. Mcintosh, A. R. et al. Recruitment of Unique Neural Systems to Support Visual Memory in Normal Aging. Current Biology Cb 9 , 1275-1278 (1999).

81. Egeth, H. \& Kahneman, D. Attention and Effort. American Journal of Psychology 88, 339 (1975).

82. León, F. R. \& Avilés, E. How altitude above sea level affects intelligence. Intelligence 58, 33-41 (2016).

83. Clarke, D. Circulation and energy metabolism of the brain. Basic neurochemistry: molecular, cellular and medical aspects, 637-669 (1999).

84. Raichle, M. E. Two views of brain function. Trends in cognitive sciences 14, 180-190 (2010).

85. Raichle, M. E. \& Gusnard, D. A. Appraising the brain's energy budget. Proceedings of the National Academy of Sciences $\mathbf{9 9}$, 10237-10239 (2002)

86. Leblond, J. \& Krnjevic, K. Hypoxic changes in hippocampal neurons. Journal of Neurophysiology 62, 1-14 (1989).

87. Mangun, G. R. \& Hillyard, S. A. Allocation of visual attention to spatial locations: Tradeoff functions for event-related brain potentials and detection performance. Perception \& Psychophysics 47, 532-550 (1990).

88. Sharma, V. K. et al. Domain specific changes in cognition at high altitude and its correlation with hyperhomocysteinemia. Plos One 9, e101448 (2014).

89. Barone, F. C. Endogenous brain protection: models, gene expression, and mechanisms. Methods Mol Med 104, 105-184 (2005).

90. Kim, J. Y. \& Yenari, M. Chapter 56 - Heat Shock Proteins and the Stress Response. Primer on Cerebrovascular Diseases, 273-275 (2017).

91. Reis, D. J., Golanov, E. V., Galea, E. \& Feinstein, D. L. Central Neurogenic Neuroprotection: Central Neural Systems That Protect the Brain from Hypoxia and Ischemia. Annals of the New York Academy of Sciences 835, 168 (1997)

92. Schmitt, K. R. L., Tong, G. \& Berger, F. Mechanisms of hypothermia-induced cell protection in the brain. Molecular \& Cellular Pediatrics 1, 7 (2014).

93. Zhang, H. C. Standardization Research on Raven's Standard Progressive Matrices in China. Acta Psychologica Sinica 21, 3-11 (1989).

94. Mesulam, M.-M. From sensation to cognition. Brain: a journal of neurology 121, 1013-1052 (1998).

95. Leslie, G., Ungerleider \& Kastner, S. Mechanisms of visual attention in the human cortex. Annual review of neuroscience 23, 315-341 (2000).

96. Bledowski, C. et al. Localizing P300 generators in visual target and distractor processing: a combined event-related potential and functional magnetic resonance imaging study. Journal of Neuroscience 24, 9353-9360 (2004).

97. Husain, M. \& Nachev, P. Space and the parietal cortex. Trends in cognitive sciences 11, 30-36 (2007).

\section{Acknowledgements}

This work was supported by the Natural Science Foundation of China (Nos 31560277, 31660274, and 31600907), the Natural Science Foundation of Tibet (2016ZR-15-3).

\section{Author Contributions}

Delong Zhang, Hailin Ma, and Ming Liu conceived the study; Hailin Ma, Xiaoyan Huang, and Delong Zhang performed the data analysis; Delong Zhang, Xiaoyan Huang, and Hailin Ma wrote the paper; Hailin Ma, Xiaoyan Huang, and Huifang Ma collected the data; and all authors reviewed the manuscript.

\section{Additional Information}

Competing Interests: The authors declare no competing interests.

Publisher's note: Springer Nature remains neutral with regard to jurisdictional claims in published maps and institutional affiliations. 
(i) Open Access This article is licensed under a Creative Commons Attribution 4.0 International License, which permits use, sharing, adaptation, distribution and reproduction in any medium or format, as long as you give appropriate credit to the original author(s) and the source, provide a link to the Creative Commons license, and indicate if changes were made. The images or other third party material in this article are included in the article's Creative Commons license, unless indicated otherwise in a credit line to the material. If material is not included in the article's Creative Commons license and your intended use is not permitted by statutory regulation or exceeds the permitted use, you will need to obtain permission directly from the copyright holder. To view a copy of this license, visit http://creativecommons.org/licenses/by/4.0/.

(C) The Author(s) 2018 